\title{
Criterios ambientales y socioeconómicos para priorizar inversiones en la red vial de Costa Rica
}

\section{Environmental and socioeconomic criteria to prioritize investments in the road network of Costa Rica}

\author{
Rodrigo Arias García \\ Consultor, Costa Rica \\ rag.1393@gmail.com
}

\author{
José David Rodríguez Morera \\ LanammeUCR, Costa Rica \\ jose.rodriguezmorera@ucr.ac.cr
}

Fecha de recepción: 07 de marzo de 2018 / Fecha de aprobación: 03 de mayo de 2018

\section{RESUMEN}

Dada la ausencia de la implementación formal de un Sistema de Gestión de Pavimentos para la Red Vial Nacional Pavimentada de Costa Rica, no han existido procesos de planificación de largo plazo sobre la inversión en los pavimentos de esa red. La presente investigación busca demostrar, además de la factibilidad de realizar planes de inversión de largo plazo (20 años) en pavimentos flexibles, también la posibilidad de utilizar índices ambientales, económicos y sociales como criterios de priorización.

Para la elaboración de planes de inversión, se estimó la condición del pavimento a través de un índice compuesto por la capacidad funcional y la capacidad estructural, en cada sección de control de la Red Vial Nacional. A cada una de estas secciones se le agregó información de tránsito, jerarquía de la ruta y otras características. Posteriormente, se estimó empleando como referencia los costos de la licitación pública 2014LN000018-0C00, el valor de cada una de las intervenciones según la ventana de operación en que se encuentre el pavimento.

Utilizando la herramienta informática Woodstock, se emplearon técnicas de optimización lineal para realizar escenarios de inversión incorporando diferentes criterios de priorización. De esta forma se emplearon el Îndice de Progreso Social, el Índice de Competitividad Cantonal y la emisión de $\mathrm{CO}_{2}$, como criterios para priorizar las inversiones en las diferentes secciones de control de la Red Vial Nacional Pavimentada de Costa Rica.

Con los resultados, es posible confirmar la factibilidad de elaborar planes de inversión de largo plazo a partir de los insumos disponibles en Costa Rica. Además, se observa que los aumentos de presupuesto tienen un mayor efecto en la rapidez con la que se alcanza una mejor condición de los pavimentos que en la condición en sí. Asimismo, la reducción de emisiones de $\mathrm{CO}_{2}$ tendrá un impacto en la condición de la red pues las intervenciones de reconstrucción son las que requieren actividades de mayor emisión de contaminantes.

PALABRAS CLAVES: gestión de pavimentos, planes de inversión; criterios de priorización; nivel de red.

\begin{abstract}
Given the absence of a formal implementation of a Pavement Management System for the National Paved Road Network of Costa Rica, there are no long-term planning processes of investments in the pavements. The research presented herein aims to demonstrate, in addition to the feasibility of perform long-term investment plans (20 years), that prioritization criteria on environmental, social and economical aspects can be included in the plan.
\end{abstract}

For the preparation of investment plans, the condition of the pavement was estimated through an index composed of functional capacity and structural capacity in each section of the National Road Network. Each of these sections contains characteristics referring to traffic, route hierarchy and others. Subsequently, costs of maintenance techniques are estimated using contract 2014LN-000018-0C00.

Through Woodstock software, linear optimization techniques were used to carry out investment scenarios incorporating different prioritization criteria. In this way, the Social Progress Index, the Cantonal Competitiveness Index and the $\mathrm{CO}_{2}$ emission are used as criteria to prioritize investments in the sections of the National Paved Road Network of Costa Rica.

With the results, it is possible to confirm the feasibility of preparing longterm investment plans based on the inputs available in Costa Rica. In addition, it is observed that the budget increases have a greater effect on the lapse with which a better condition of the pavements is achieved than in the condition itself. Likewise, the reduction of $\mathrm{CO}_{2}$ emissions will have an impact on the condition of the network, since reconstruction interventions are those that require activities with a higher emission of pollutants.

KEY WORDS: pavement management; investment plan; prioritization criteria; network level. 


\section{INTRODUCCIÓN}

El presente artículo muestra los resultados de incorporación criterios económicos, sociales y ambientales en la priorización de inversiones en una red vial pavimentada. La gestión de redes viales pavimentadas constituye un reto para las instituciones y agencias de transporte. Las restricciones presupuestarias, las necesidades de inversión de acuerdo a la condición de los pavimentos e incluso las expectativas del público son las variables principales con las cuales las instituciones deben buscar la mejor forma de utilizar los recursos disponibles para el mantenimiento y desarrollo de las redes viales.

Sin embargo, la importancia alcanzada por los temas ambientales debido a las amenazas planteadas por fenómenos asociados al cambio climático, así como la dinámica de la participación ciudadana en los asuntos públicos hace que las políticas de mantenimiento de infraestructura tenga un foco más amplio en sus consideraciones. Por esta razón, este trabajo ensaya la incorporación de criterios de priorización relacionados con estas áreas, todo dentro del marco de la filosofía de gestión de pavimentos (basada también en la gestión de activos de transporte-transportation asset management-).

Tal como la cita la Dirección de Vialidad del Ministerio de Obras Públicas de Chile (2014), la gestión vial es "el conjunto de operaciones que tienen por objetivo conservar adecuadamente y por un determinado período, una carretera o una red vial en condiciones apropiadas de seguridad, comodidad y capacidad estructural, bajo las condiciones ambientales locales. Lo anterior debe ejecutarse minimizando los requerimientos financieros, con el máximo beneficio social posible y con los menores impactos ambientales negativos".

Específicamente, un Sistema de Gestión de Pavimentos es, según Solminihac (1998), "el conjunto de operaciones que tienen como objetivo conservar por un período de tiempo las condiciones de seguridad, comodidad, y capacidad estructural adecuadas para la circulación, soportando las condiciones climáticas y de entorno de la zona en que se ubica la vía en cuestión. Todo lo anterior minimizando los costos monetario, social y ecológico".

De acuerdo con la Asociación de Transportes de Canadá (1997), el objetivo es cumplido mediante la comparación de alternativas de inversión tanto a nivel de red, como de proyecto, los cuales son los dos niveles que componen un Sistema de Gestión de Pavimentos (Haas, 1993). En la siguiente Figura 1 se observa la estructura típica dentro de un Sistema de Gestión de Pavimentos.

\section{NIVEL DE RED}

- Metas y políticas

- Recolección y procesamiento de datos

- Criterios de priorización para Mantenimiento y Rehabilitación

- Modelos de deterioros

- Comparación de escenarios de inversión

- Restricciones presupuestarias

- Planeamiento y programación

NIVEL DE PROYECTO

- Recolección de datos en sitio

- Análisis económico e ingenieril del proyecto

- $\quad$ Selección de mejor alternativa

- Plan detallado de ejecución

- Inspección de las obras

- Control de calidad de los materiales

Figura 1. Estructura típica de un Sistema de Gestión de Pavimentos

Dentro estos procesos de gestión de pavimentos, el plan de inversiones es fundamental pues es la "brújula" que dirige los esfuerzos en la dirección correcta para concretar los objetivos planteados. Los criterios de priorización de las inversiones son el mecanismo para concretar las intenciones de la inversión más allá de mejorar la condición de la superficie de rodadura en una red vial.

Utilizando el caso de la RVN pavimentada de Costa Rica, se determinó la condición del pavimento a partir del Índice de Regularidad Internacional (IRI) y deflectometría medidos por el Laboratorio Nacional de Materiales y Modelos Estructurales (LanammeUCR). Además, se estimaron los costos asociados a su ventana de operación de mantenimiento. Posterior a esto, usando herramientas informáticas de optimización lineal, se incorporan en esta investigación criterios económicos, sociales y ambientales para modelar la priorización de las inversiones en la Red Vial Nacional pavimentada de Costa Rica. 


\section{ANTECEDENTES}

La metodología utilizada para el desarrollo del planes de inversión que siguió esta investigación tiene su primer antecedente en la tesis de grado "Plan de Inversiones a Nivel Estratégico para la Zona 1-9 de Conservación Vial de CONAVI" (Chaverri y Madrigal, 2011). En ese trabajo se estableció el procedimiento de modelos de optimización lineal para planes de inversión a largo plazo. Además se creó la subrutina del modelo de optimización en el programa de cómputo Woodstock.

Posteriormente, esta metodología se aplicó a la totalidad de la RVN pavimentada en la investigación "Plan de inversión a nivel estratégico en pavimentos flexibles de la Red Vial Nacional" (Rodríguez y Chaverri, 2012). En ese trabajo se desarrollaron diferentes escenarios de inversión para desarrollar un plan de inversiones óptimo para el mantenimiento de la RVN. Para la priorización de la inversión se emplearon criterios como el tránsito promedio diario y la pertenencia de la ruta a la red vial estratégica del país. (Figura 2)

\section{OBJETIVOS}

\section{Objetivo General}

Desarrollar un plan de inversiones a nivel de red para pavimentos flexibles de la Red Vial Nacional que incorpore criterios de priorización relacionados con aspectos ambientales, sociales y económicos de acuerdo con insumos disponibles en el país.

\section{Objetivos Específicos}

- Determinar la condición del pavimento a través de un índice compuesto por la capacidad funcional y estructural para las secciones de control de los pavimentos flexibles de la Red Vial Nacional, según los parámetros IRI y FWD medidos en la evaluación técnica de la RVN del LanammeUCR que comprende los años 2014 - 2015.

- Explorar el uso de índices o criterios ambientales, sociales y económicos que puedan ser incorporados en la priorización de inversiones en pavimentos flexibles de la Red Vial Nacional.

- Realizar diferentes escenarios de inversión para la RVN considerando criterios ambientales, sociales y económicos, mediante la aplicación de técnicas de optimización lineal.

\section{METODOLOGÍA}

La metodología de la presente investigación se muestra en la Figura 2. Como se observa, la metodología se conforma de tres principales etapas: recolección de la información, procesamiento de la información sobre la RVN pavimentada de Costa Rica y estimación de la condición del pavimento según sección de control; y por último, utilización de la herramienta informática Woodstock para optimización lineal.

\section{Base de Datos Sobre la RVN Pavimentada}

La RVN pavimentada de Costa Rica cuenta con amplia información sobre la condición de sus pavimentos. La evaluación bienal que realiza el LanammeUCR mide índices como el IRI, deflectometría y el Grip Number (resistencia al deslizamiento). En este caso, la información de la condición funcional (IRI) y la (deflectometría) de la evaluación 2014-2015 fueron utilizadas para la determinación de un índice de condición del pavimento (PCI) en cada sección de control de la red vial analizada.

Además de la condición del pavimentos, a cada sección de control se le asignó la información del Índice de Progreso Social (CLACDS, 2016) e Índice de Competitividad Cantonal (Castro, 2015) correspondiente a la zona geográfica que cada ruta nacional atraviesa. Con esto se buscó crear información relacionada con aspectos sociales y económicos.

Por último, para incorporar el criterio ambiental se obtuvieron los valores de emisión de $\mathrm{CO}_{2}$ de tratamientos de mantenimiento vial similares a los empleados en la modelación de las inversiones en la RVN realizaras en este trabajo. Así, se utilizó como referencia los resultados obtenidos por Chehovits y Galehouse (2010) para diferentes actividades de mantenimiento de los pavimentos.

\section{Estimación de la Condición de los Pavimentos}

Utilizando la información de la regularidad superficial y de la capacidad de los pavimentos flexibles de la RVN de Costa Rica, se procedió a estimar su condición mediante un índice compuesto por ambas mediciones. Este índice PCI (Pavement Condition Index) es calculado para cada sección de control la RVN asignando un peso de $50 \%$ a cada uno de estos componentes (Mata, 2010), luego de haber procesado los datos provenientes de la evaluación bienal.

Una vez estimada la condición de cada pavimento, ésta se clasificó en categorías (Muy Bueno, Bueno, Regular, Pobre y Muy Pobre) cuya condición está asociada a ventanas de operación y sus respectivos costos (Preservación, Rehabilitación Menor, 
Rehabilitación Mayor, Reconstrucción Menor y Reconstrucción Total). Las curvas de deterioro utilizadas para la modelación son las propuestas por Amador (2011) citadas por Rodríguez (2012).

\section{Optimización Lineal para el Plan de Inversiones}

A través de la herramienta informática Woodstock de Remsoft Inc. se procedió a realizar escenarios de inversión en los pavimentos flexibles de la RVN de Costa Rica. En este caso se ensayaron escenarios que utilizaron como criterios de priorización los índices de progreso social y de competitividad cantonal, además de los escenarios típicos correspondientes a restricción presupuestaria (función objetivo minimizar costos y maximizar condición del pavimento), restricción de plazo para conseguir metas de condición (función objetivo eliminar pavimentos en estado "muy pobre").

En el caso del aspecto ambiental, éste fue modelado incorporando la emisión de $\mathrm{CO}_{2}$ de cada tratamiento como un costo ambiental. Por lo tanto, el objetivo en la modelación fue la reducción de la cantidad de emisiones de $\mathrm{CO}_{2}$.

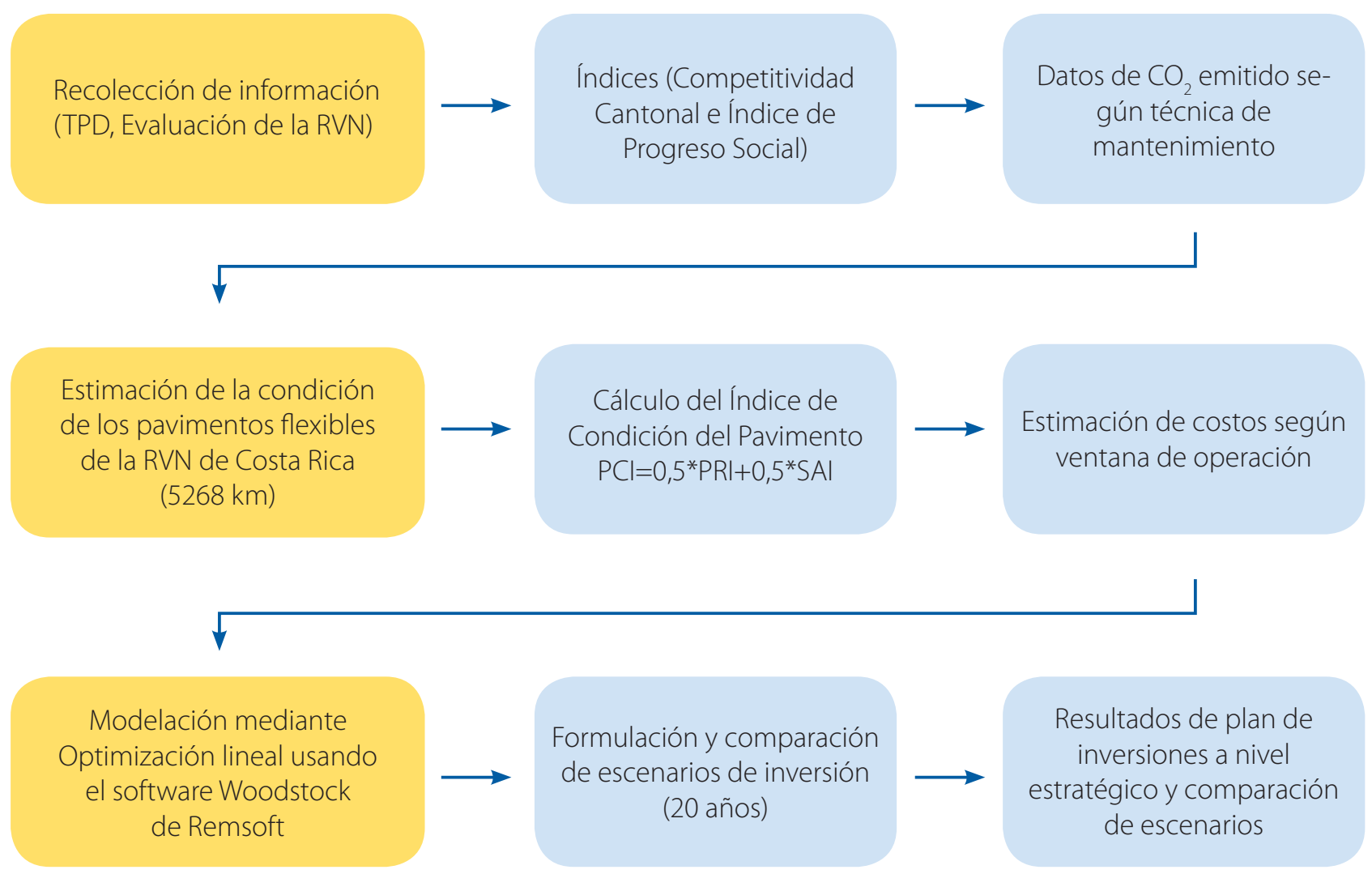

Figura 2. Metodología de trabajo para la incorporación de criterios ambientales, sociales y económicos en la priorización de inversiones en pavimentos flexibles a nivel de red 


\section{RESULTADOS}

\section{Estimación de la Condición del Pavimento (PCI)}

El Índice de Condición del Pavimento es el indicador utilizado para determinar la condición del pavimento en esta investigación. Este índice se puede obtener a partir de las mediciones realizadas en las evaluaciones técnicas, al relacionar la deflectometría (FWD) con la regularidad superficial (IRI). Según Chaverri \& Madrigal (2011) este índice puede calcularse de la siguiente manera:

$$
P C I=k_{1} * S A I+k_{2} * P R I
$$

Donde, $S A I$ = índice de adecuación estructural derivada a partir de los datos del deflectómetro de impacto FWD, $P R I=$ índice de regularidad superficial del pavimento derivado a partir del IRI, $\mathrm{k}_{1}$ $\mathrm{y}_{2}$ son factores de peso relativo en el índice. Los valores de PRI para cada sección del pavimento pueden ser calculados mediante una función normalizadora que traduce cada valor de IRI en una escala de 0 - 100 (Chaverri \& Madrigal, 2011):

$$
P R I_{j}=100\left(\frac{I R I_{\text {max }}-I R I_{j}}{I R I_{\text {max }}-I R I_{\text {min }}}\right)
$$

En la anterior ecuación, PRIj es el índice de regularidad superficial del pavimento de la j-ésima sección de una carretera (tramo homogéneo), IRIj es el valor medido de IRI para la j-ésima sección, IRI ${ }_{\text {max }}$ e IRI $_{\text {min }}$ son los valores máximos y mínimos de IRI para toda la Red en estudio, para un nivel de confianza del 98\%.
El índice de adecuación estructural (SAI) provee una medida de la capacidad de carga o resistencia del pavimento. La ecuación (4) ha sido desarrollada al tomar la resistencia del pavimento expresada por el parámetro base de deflexiones conocido como " $A R E A$ " (ecuación 3) normalizado por la deflexión en el centro de carga, $D_{0}$ (la cual se corrige por temperatura) y luego se convierte en un índice escalado entre 0 - 100 para la formulación del PCI.

$A R E A=150 *\left(\frac{D_{0}+2 D_{1}+2 D_{2}+D_{3}}{D_{0}}\right)$

Donde, $\mathrm{D}_{0}, \mathrm{D}_{1}, \mathrm{D}_{2}, \mathrm{D}_{3}$ son deflexiones medidas desde el centro del plato de carga del deflectómetro de impacto (FWD) de las ordenadas a $0 \mathrm{~mm}, 300 \mathrm{~mm}, 600 \mathrm{~mm}$ y $900 \mathrm{~mm}$ respectivamente.

$S A I_{j}=100\left(\frac{A R E A_{j}-A R E A_{\text {min }}}{A R E A_{\text {max }}-A R E A_{\text {min }}}\right)$

En esta ecuación, $S A I j$ es el índice de adecuación estructural para el j-ésimo tramo homogéneo, $A R E A$ se define como se muestra en la ecuación (3), $A R E A_{\max }$ y $A R E A_{\min }$ son los valores máximos y mínimos del $A R E A$ a nivel de toda la red, con un intervalo de confianza del $98 \%$.

Con el resultado del valor del PCI se clasifica la condición del pavimento, como se ve el Cuadro 1, y de acuerdo a esto se define el tipo de tratamiento que debe recibir el pavimento (ventana de operación). Los rangos de PCI fueron tomados de la experiencia del Departamento de Transportes de New Brunswick en Canadá (2002) reseñada por Madrigal y Chaverri (2011).

\section{Cuadro 1. Escala de clasificación del PCl y su ventana de operación.}

\begin{tabular}{|c|c|c|c|c|c|}
\hline \multirow{2}{*}{ Condición } & Rang0 & \multicolumn{2}{|c|}{ Costo según Tránsito (USD) } \\
\hline Muy Bueno & $80-100$ & $\begin{array}{c}\text { Preservación (sobrecapa no } \\
\text { estructural 4cm - SNE-) }\end{array}$ & \multicolumn{2}{|c|}{$\$ 27.767,85$} \\
\hline Bueno & $60-80$ & $\begin{array}{c}\text { Rehabilitación menor } \\
\text { (Sobrecapa estructural) }\end{array}$ & $\$ 34.709,81$ & $\$ 62.477,66$ \\
\hline Regular & $40-60$ & $\begin{array}{c}\text { Rehabilitación mayor } \\
\text { (Sobrecapa estructural) }\end{array}$ & $\$ 69.419,62$ & $\$ 104.129,44$ & $\$ 131.897,29$ \\
\hline Pobre & $25-40$ & $\begin{array}{c}\text { Reconstrucción menor } \\
\text { (Base Estabilizada + Capa Asfáltica) }\end{array}$ & $\$ 154.669,23$ & $\$ 164.856,87$ & $\$ 191.933,43$ \\
\hline Muy Pobre & $0-25$ & $\begin{array}{c}\text { Reconstrucción total } \\
\text { (Nueva estructura) }\end{array}$ & $\$ 190.258,10$ & $\$ 230.084,11$ & $\$ 241.144,23$ \\
\hline
\end{tabular}

1 Rangos establecidos por New Brunswick Department of Transportation, Canadá (2002). 
Los costos utilizados corresponden a los establecidos en los contratos de conservación vial perteneciente a la licitación pública 2014LN-000018-0C00 en Costa Rica. Se empleó el valor promedio para los ítems con precios unitarios diferentes por zona geográfica. En el Cuadro 1 se muestra el costo de las intervenciones por kilómetro de carril según la intensidad de tránsito que presenten. Para estimar los costos se empleó un ancho de carril promedio de 3,33 m obtenido de los registros para la RVN pavimentada de Costa Rica.

\section{Índices para la Priorización de Inversiones en el Pavimento de la RVN de Costa Rica}

\section{Índice de Competitividad Cantonal}

El Îndice de Competitividad Cantonal (ICC) evalúa la capacidad productiva de cada cantón, y demás factores que influyan en su desarrollo. Asimismo, permite identificar los campos en los que se requieren intervenciones institucionales o políticas públicas (Castro, 2015). Se definen cinco categorías del ICC con el fin de distribuir los cantones y visualizar su evolución con respecto a la última medición: muy alta, alta, media, baja y muy baja.

\section{Índice de Progreso Social}

El Índice de Progreso Social (IPS) permite evaluar la eficacia con la cual el éxito económico de un país o región se traduce en progreso social. Para realizar la medición del progreso social, el IPS considera tres dimensiones: necesidades humanas básicas, fundamentos de bienestar, y oportunidades. A su vez, estas tres dimensiones se subdividen en 12 componentes, que agrupan 46 indicadores (CLACDS, 2016). Se clasifica el desempeño de cada cantón en: bueno, regular y malo.

\section{Costo Ambiental de las Intervenciones en los Pavimentos Flexibles}

Para determinar el costo ambiental (entendido como emisiones de $\mathrm{CO}_{2}$ ) de las intervenciones se emplearon los valores de la investigación de Chehovits y Galehouse (2010). En el Cuadro 2 se muestra la emisión de $\mathrm{CO}_{2}$ de cada uno de los tratamientos, por kilómetro de carril, utilizados en la presente investigación.

\section{Escenarios de Inversión a Largo Plazo en la RVN Pavimentada de Costa Rica}

El objetivo planteado con la elaboración de los escenarios de inversión es proyectar la evolución de la condición de los pavimentos en el largo plazo (20 años) ante diferentes estrategias. La ventaja de los análisis de largo plazo, bajo los principios de un Sistema de Gestión de Pavimentos, es que a través de la comparación de alternativas de inversión es posible disminuir la incertidumbre sobre la eficacia de las políticas de mantenimiento aplicadas.

El plazo de análisis está relacionado con la recomendación de analizar, a nivel de red o estratégico, el impacto de las decisiones en un período de al menos el doble del período de vida útil del activo. Por esta razón, en el caso de los pavimentos se recomienda mantener un plazo de análisis de al menos 20 años.

En la Figura 3 se muestra la salida para el escenario "Optimizar puro" que busca maximizar la condición de los pavimentos de la RVN de Costa Rica, utilizando una restricción presupuestaria de USD \$135 millones, monto semejante al que invierta la agencia gubernamental encargada (Consejo Nacional de Vialidad). Como se observa, a la mitad del período, los pavimentos en Muy Pobre, Pobre y Regular condición son eliminados si se siguen las estrategias descritas en el Cuadro 1.

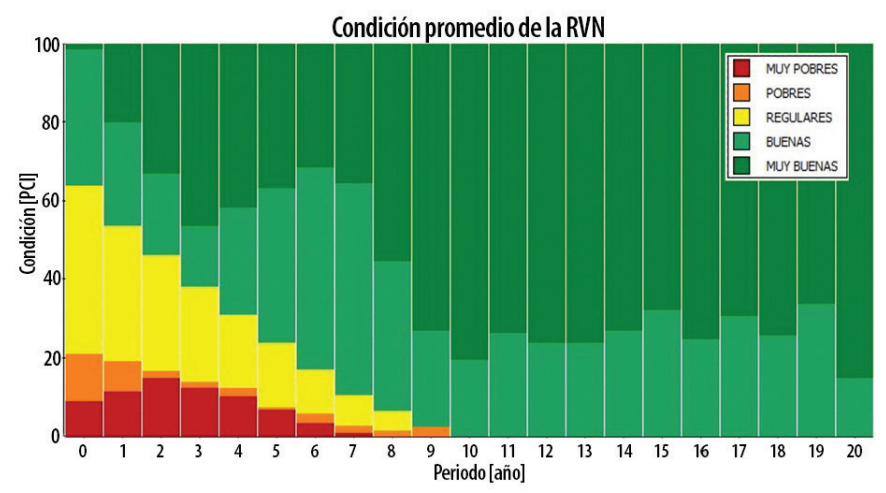

Figura 3. Evolución de la condición de la RVN pavimentada de Costa Rica al maximizar el $\mathrm{PCI}$ con un presupuesto anual de USD \$135 millones crecimiento anual de 5\%

Cuadro 2. Emisión de $\mathrm{CO}_{2}$ por kilómetro de carril según intensidad de tránsito

\begin{tabular}{|c|c|c|c|c|c|}
\hline \multirow{2}{*}{ Clasificación } & \multirow{2}{*}{$\mathrm{PCl}$} & \multirow{2}{*}{ Tratamiento } & \multicolumn{3}{|c|}{ Intensidad de tránsito } \\
\hline & & & Bajo & Medio & Alto \\
\hline Muy bueno & $80-100$ & SNE & \multicolumn{3}{|c|}{16237.08} \\
\hline Bueno & $60-80$ & Reh. Menor & 20296.35 & 36533.43 & 52770.51 \\
\hline Regular & $40-60$ & Reh. Mayor & 40592.70 & 60889.05 & 77126.13 \\
\hline Pobre & $25-40$ & Rec. Menor & 87299.28 & 91425.15 & 109630.26 \\
\hline Muy pobre & $0-25$ & Rec. Total & 115893.99 & 140066.46 & 145504.35 \\
\hline
\end{tabular}

Fuente: Chehovits y Galehouse (2010) 
Otro de los escenarios de inversión típicos a analizar son los que modelan el efecto de la variación en el propio monto del presupuesto. En la Figura 4 se observa el resultado de la comparación, teniendo como restricciones que RVN estratégica (rutas principales definidas por el Ministerio de Obras Públicas de Costa Rica) alcanza un PCI promedio de 65 al primer año, y un PCI promedio mínimo de 80 (condición muy buena) al año 5 .

Como se puede ver, incrementar el presupuesto en un 50\% (presupuesto inicial de $\$ 202,5$ millones) influye poco en el estado final de la red. El efecto de incrementar el presupuesto se manifiesta en el tiempo requerido para obtener una condición muy buena de la red vial, pues al aumentar el presupuesto un 50\% se obtiene un PCI mayor a 80 en la mitad del tiempo (Figura 4). Este análisis es importante a la hora de decidir estrategias frente otras necesidades en una Administración.

Evolución del $\mathrm{PCl}$ de la RVN según variación en el presupuesto

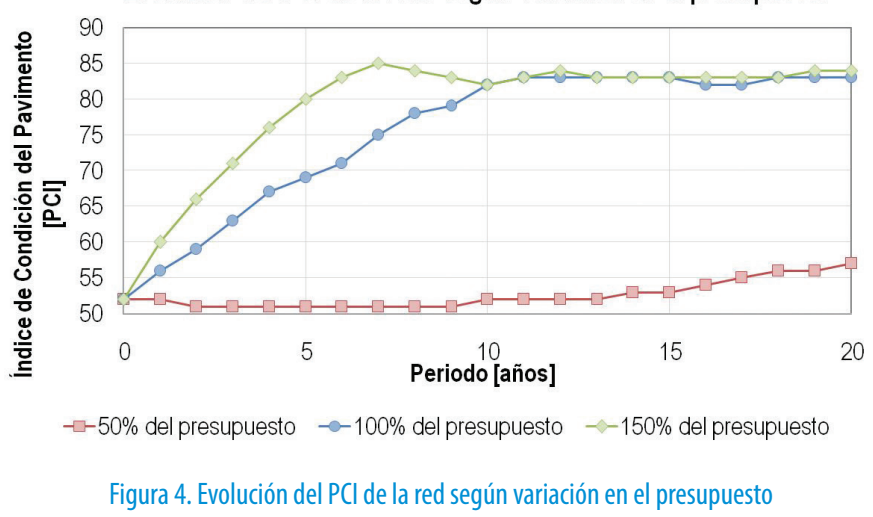

\section{Incorporación de Criterios de Priorización Ambientales,} Económicos y Sociales

En estos escenarios se busca maximizar la condición promedio de la RVN mientras se realiza la priorización de las secciones a intervenir de acuerdo a diferentes criterios socioeconómicos, por ejemplo: se prioriza la RVN estratégica, las secciones con un elevado tránsito promedio diario, y las secciones que se encuentren en cantones con un bajo Índice de Competitividad Cantonal o Índice de Progreso Social. En la Figura 5 se muestra la evolución de la condición de la RVN de acuerdo al escenario de inversión incluido.

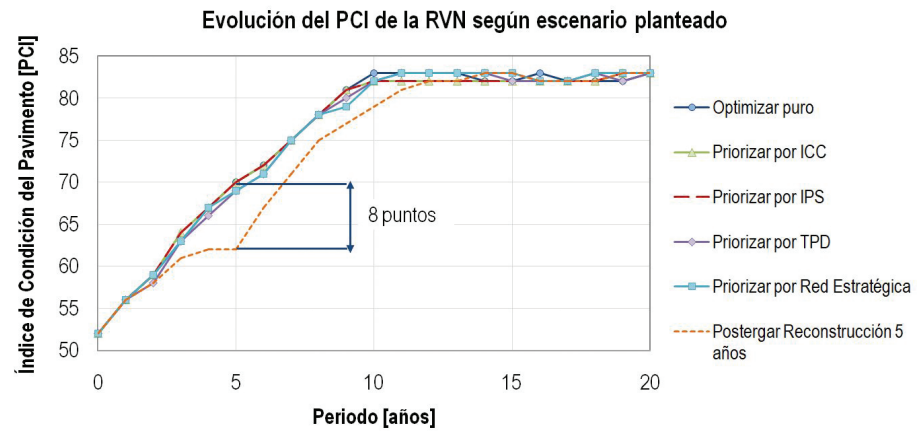

Figura 5. Evolución de la condición de la RVN según el escenario propuesto
Debido a las restricciones utilizadas, no existe diferencia significativa en la condición promedio final de la red vial según el criterio de priorización seleccionado. Sin embargo, si hay diferencia en el tiempo requerido para alcanzar un valor promedio bueno. En los distintos escenarios modelados, la RVN finaliza en "Muy Buena" condición $(\mathrm{PCI}=83)$.

El escenario "Postergar Reconstrucción 5 años" surge de la necesidad de modelar los efectos burocráticos en el arranque de los proyectos. Es decir, si las reconstrucciones se postergan un período de 5 años mientras se realizan los procesos administrativos necesarios, el costo para el usuario será una brecha de hasta 8 puntos promedio en la condición de las rutas que utiliza.

\section{Criterio Ambiental}

Con este escenario se busca reducir la cantidad de emisiones de $\mathrm{CO}_{2}$ de las distintas intervenciones, estableciendo requisitos de condición mínimos al pavimento (restricciones en la optimización lineal). Por ejemplo, una condición promedio "Muy Buena" de la RVN estratégica, o la eliminación de las secciones en pobre o muy pobre condición.

Como el objetivo de este escenario no es optimizar la condición de la red sino minimizar la emisión de $\mathrm{CO}_{2}$, la condición final de la red vial es inferior al escenario "Optimizar puro", pues únicamente busca cumplir con las restricciones establecidas. Esto se puede observar en la Figura 6 que compara ambos PCI promedios y en la Figura 7 que muestra las cantidades de pavimentos según la condición. En esta última, se observa que las condiciones Pobre y Regulares no se logran eliminar del todo.

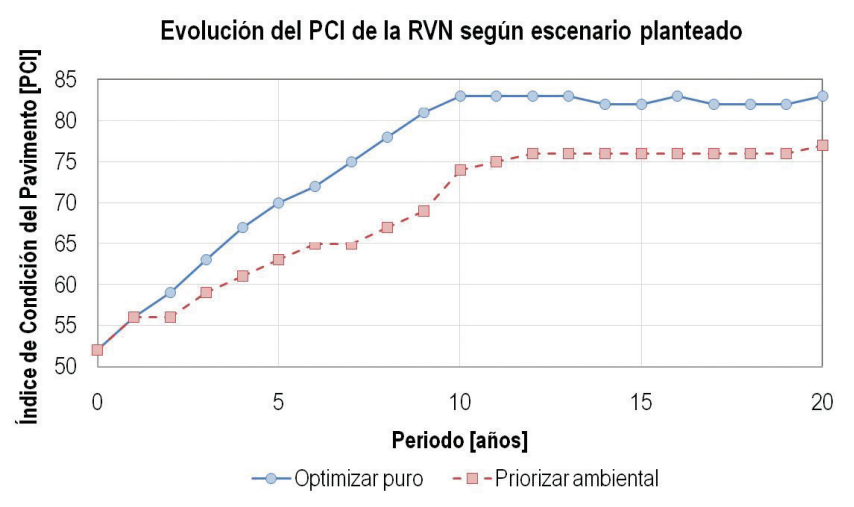

Figura 6. Comparación de la condición de la RVN para los escenarios "Optimizar puro" $y$ "Priorizar ambiental" 


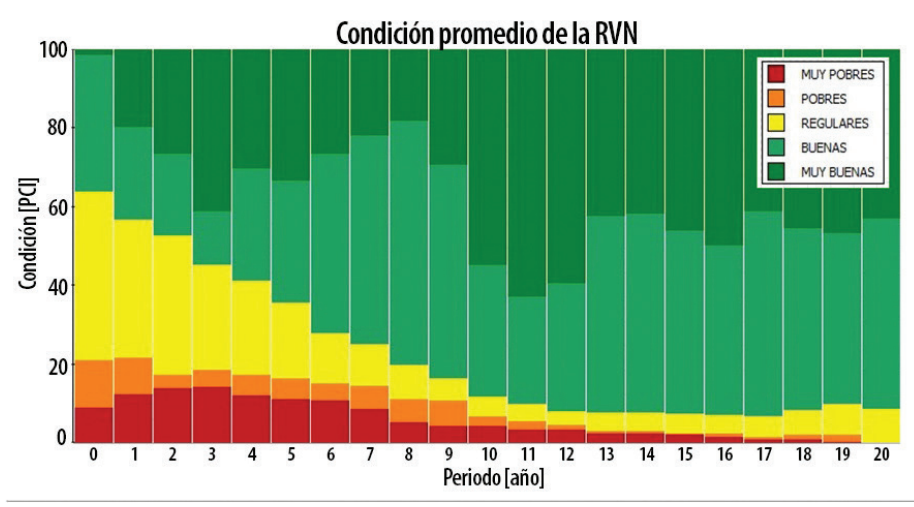

Figura 7. Evolución de la condición de la RVN pavimentada de Costa Rica al minimizar la emisión de $C 22$ con un presupuesto anual de USD \$135 millones crecimiento anual de $5 \%$

A pesar de que el escenario solo busca cumplir con las restricciones propuestas al menor coste ambiental posible, la red vial finaliza en buena condición (PCI 6 puntos por debajo del escenario "Optimizar puro"), requiriendo en promedio un 32\% menos de inversión anual y emitiendo un 31\% menos de contaminantes.

En resumen, tener la posibilidad de realizar análisis de este tipo entre distintos escenarios representa una oportunidad importante para mejorar el diseño de políticas públicas. En el análisis de largo plazo que disminuye la incertidumbre sobre el impacto de la inversión en los pavimentos, se encuentra la diferencia entre ejecutar un presupuesto en estrategias inadecuadas e invertir un presupuesto de forma óptima.

De esta forma, las agencias encargadas pueden conocer con antelación el estado final del activo, la inversión requerida y la cantidad de $\mathrm{CO}_{2}$ emitida en su mantenimiento, lo que permite alinear las políticas de inversión con las metas planteadas por parte de un gobierno, por ejemplo en materia ambiental.

\section{CONCLUSIONES}

- Todo plan de inversiones en pavimentos debe seguir los principios de un Sistema de Gestión de Carreteras para obtener resultados óptimos, es decir, se debe aplicar el tratamiento adecuado, en la sección apropiada, en el tiempo indicado.

- Se obtiene un mismo resultado en la condición promedio final del pavimento al realizar una optimización pura (sin ninguna restricción socioeconómica) que si se realiza la priorización de inversiones considerando aspectos socioeconómicos debido a incluir restricciones similares. Al incluir criterios sociales, económicos y ambientales en la toma de decisiones se invertirá en zonas donde se pueden lograr externalidades positivas debido a la inversión realizada.

- Un incremento en el presupuesto asignado para atender la red vial no representa una gran mejoría en la condición final de la red, sino un adelanto en el tiempo necesario para alcanzar un buen estado. Esto representa una mejora en el desarrollo socioeconómico de una región o país, pues la condición del patrimonio vial se encuentra relacionada con el gasto de combustible y los egresos por mantenimiento de los vehículos de parte de los usuarios. De la misma forma, se garantizan condiciones de seguridad y confort para los usuarios en la carretera en un menor tiempo. Por su parte, una reducción en el presupuesto si afecta negativamente la condición final de la red.

- Se debe encontrar un equilibrio entre condición de la red vial, presupuesto y emisión de $\mathrm{CO}_{2}$, pues los tratamientos más costosos económica y ambientalmente son aquellos que permiten levantar la condición de las secciones en pobre o muy pobre estado, de ahí la importancia de un enfoque preventivo, en lugar de uno reactivo.

\section{REFERENCIAS}

1. Amador, L., Chaverri, J., \& Rodríguez, J. (2012). A Pavement Management System for Costa Rica: a case study. San José: LanammeUCR.

2. Castro, A. (2015). Fichero cantonal 2016. San José: Instituto de Formación y Estudios en Democracia.

3. Chaverri, J., \& Madrigal, D. (2011). Modelos de optimización lineal para realizar planes de inversiones de largo plazo en una red de pavimentos. Infraestructura Vial, 22-30.

4. Chehovits, J., \& Galehouse, L. (2010). Energy usage and greenhouse gas emissions of pavement preservation processes for asphalt concrete pavements. California: 1st International Conference of Pavement Preservation.

5. CLACDS. (2016). Índice de Progreso Social 2016. San José: INCAE Business School.

6. Dirección de Vialidad de Chile. (2014). Manual de Carreteras: Volumen N 7 Mantenimiento Vial. Santiago, Chile: Ministerio de Obras Públicas.

7. Mata, D. (2010). Índices de Condición de Pavimentos Flexibles. Trabajo Final de Graduación, Escuela de Ingeniería Civil, Universidad de Costa Rica.

8. Rodríguez, J. D. (2012). Plan de inversión a nivel estratégico en pavimentos flexibles de la Red Vial Nacional. San José, C. R.: TFG: Escuela de Ingeniería Civil, UCR.

9. $\quad$ Hass, R.; Hudson, W.R.; Zaniewski, J. (1993). Modern Pavement Management. R.E. Krieger Publishing Company, Florida

10. Solminihac, H. (1998). Gestión de Infraestructura Vial. Chile: Ediciones Universidad Católica de Chile. 